\title{
The Dissociation Constants of Some Disubstituted Anilines and Phenols in Aqueous Solution at $25{ }^{\circ} \mathrm{C}$
}

\author{
R. A. Robinson \\ Institute for Materials Research, National Bureau of Standards, Washington, D.C. 20234 \\ (February 21, 1967)

\begin{abstract}
The dissociation constants of six disubstituted anilines and of five disubstituted phenols in aqueous solution at $25{ }^{\circ} \mathrm{C}$ have been measured using the spectrophotometric method. Consideration is given to the extent to which these dissociation constants can be predicted from corresponding values for monosubstituted anilines and phenols. In addition, the question as to whether the $p K$ value of a substituted aniline can be predicted from the $p K$ value of a phenol with substituents in the same positions is also investigated.
\end{abstract}

Key Words: Dissociation constant, substituted anilines, substituted phenols.

\section{Introduction}

In an earlier paper [1] ${ }^{1}$ the results of measurements of the $p K$ values of the six dichloroanilines and the six dichlorophenols in aqueous solution at $25^{\circ} \mathrm{C}$ have been reported. The conclusions drawn from this work can be summarized briefly in two statements. In the first place, the $p K$ value of any one of ten dichlorosubstituted compounds' (2,6-dichloroaniline and 2,6-dichlorophenol being exceptions) could be predicted knowing the $p K$ values of the monosubstituted chlorocompounds. This utilizes an additivity rule which can be illustrated as follows; the $p K$ value of phenol itself is 9.998, that of $m$-chlorophenol is 9.130 and that of $p$-chlorophenol is 9.418 [2]. Thus, $m$-Cl substitution lowers the $p K$ value of phenol by 0.868 and $p$-Cl substitution by 0.580 . If the substituent effects were strictly additive, 3,4-dichloro substitution should lower the value of $p K$ by 1.448 and the $p K$ value of 3,4-dichlorophenol should be 8.550 . The observed value is 8.585 , differing from the calculated by only 0.035 . The additivity rule predicts $p K$ values with an accuracy which varies from 2,4-dichloroaniline $\left(p K_{\text {obs }}=2.02, \quad p K_{\text {calc }}=2.03\right) \quad$ to 2,3 -dichloroaniline $\left(p K_{\text {obs }}=1.76, \quad p K_{\text {calc }}=1.92\right)$. Only in the case of 2,6-dichloroaniline and 2,6-dichlorophenol did the additivity rule fail to predict the observed value within these limits, the difference between observed and calculated $p K$ values being 0.28 in the case of 2,6 -dichloroaniline and 0.27 in the case of 2,6-dichlorophenol. In these two instances we have compounds with three substituent groups in adjacent positions, $\mathrm{Cl}, \mathrm{NH}_{2}$ (or

${ }^{1}$ Figures in brackets indicate the literature references at the end of this paper.
$\mathrm{OH}), \mathrm{Cl}$, and it is not surprising that there should be steric effects which interfere with the additivity relation.

Secondly, it was found that a linear relationship existed between the $p K$ value of a dichloroaniline, $p K_{A}$, and the $p K$ value of the corresponding dichlorophenol, $p K_{P}$. This could be expressed by the equation

$$
p K_{A}=-9.047+1.401 p K_{P} .
$$

For example, it was found that $p K_{P}$ was 6.791 for 2,6-dichlorophenol, whence this equation gives $p K_{A}=0.467$. The experimental value is 0.422 . It should be noted that this relationship holds for 2,6-disubstituted compounds, although the additivity rule, mentioned above, fails in this instance.

These conclusions are valid for a particular class of disubstituted anilines and phenols, the dichloro com-ı pounds. It is of interest, however, to inquire whether they have application to a wider group of disubstituted anilines and phenols. For this reason, measure-1 ments have now been made on a group of disubstituted compounds in which the substituent groups are $-\mathrm{Br},-\mathrm{Cl},-\mathrm{CH}_{3}$, and $-\mathrm{NO}_{2}$.

\section{Experimental Procedure}

\subsection{Materials}

4-Chloro-2-methylaniline hydrochloride was recrystallized twice from 80 percent ethanol, Cl found 19.92 percent, calc 19.91 percent, $\epsilon_{2} 2,510$ at $291 \mathrm{~m} \mu$ in alkaline solution. 2,4-Dibromoaniline was recrystallized first from heptane and then from a mixture of 
heptane and petroleum ether, $\mathrm{mp} 80^{\circ} \mathrm{C}, \epsilon_{2} 2,160$ at $300 \mathrm{~m} \mu$ in alkaline solution, $\epsilon_{1} 280$ at $273 \mathrm{~m} \mu$ and 200 at $280 \mathrm{~m} \mu$ in acid solution. 2,6-Dibromoaniline was recrystallized twice from heptane, $\mathrm{mp} 83.5^{\circ} \mathrm{C}, \epsilon_{2} 2,550$ at $294 \mathrm{~m} \mu, \epsilon_{1} 350$ at $272 \mathrm{~m} \mu$ and 370 at $279 \mathrm{~m} \mu$. 2-Methyl-5-nitroaniline was recrystallized once from benzene, $\mathrm{mp} 108{ }^{\circ} \mathrm{C}, \epsilon_{2} 2,060$ at $355 \mathrm{~m} \mu$. 4-Methyl-2nitroaniline was recrystallized once from benzene, mp $117.5{ }^{\circ} \mathrm{C}, \epsilon_{2} 4,230$ at $427 \mathrm{~m} \mu$. 4-Methyl-3-nitroaniline was recrystallized twice from benzene, $\mathrm{mp}$ $79.5{ }^{\circ} \mathrm{C}, \epsilon_{2} 1,580$ at $357 \mathrm{~m} \mu$. 4-Chloro-2-methylphenol was recrystallized three times from petroleum ether, mp $47.5-48{ }^{\circ} \mathrm{C} \epsilon_{2} 3,030$ at $300 \mathrm{~m} \mu, \epsilon_{1} 1,680$ at $281 \mathrm{~m} \mu$, and isosbestic points $\epsilon 1,010$ at $271 \mathrm{~m} \mu$ and 1,600 at $284 \mathrm{~m} \mu$. 2,4-Dibromophenol was recrystallized three times from heptane, mp $36{ }^{\circ} \mathrm{C}, \epsilon_{2} 3,700$ at $309 \mathrm{~m} \mu, \epsilon_{1} 2,090$ at $286 \mathrm{~m} \mu, \epsilon 1,280$ at $274 \mathrm{~m} \mu$ and 1,970 at $291 \mathrm{~m} \mu$. 2,6-Dibromophenol was recrystallized twice from petroleum ether, $\mathrm{mp} 56^{\circ} \mathrm{C}, \epsilon_{2} 5,250$ at $304 \mathrm{~m} \mu, \epsilon_{1} 2,170$ at $276 \mathrm{~m} \mu$ and 2,170 at $284 \mathrm{~m} \mu$, $\epsilon 1,130$ at $269 \mathrm{~m} \mu$ and 2,160 at $284 \mathrm{~m} \mu$. 2-Methyl-4nitrophenol was recrystallized twice from water, $\mathrm{mp}$ $96{ }^{\circ} \mathrm{C}, \epsilon_{2} 13,310$ at $414 \mathrm{~m} \mu, \epsilon_{1} 6,340$ at $327 \mathrm{~m} \mu, \epsilon 3,510$ at $358 \mathrm{~m} \mu$. 4-Methyl-3-nitrophenol was recrystallized from benzene and then from a mixture of heptane and petroleum ether, $\mathrm{mp} 77.5-78{ }^{\circ} \mathrm{C}, \epsilon_{2} 1,410$ at $390 \mathrm{~m} \mu$, $\epsilon_{1} 2,050$ at $340 \mathrm{~m} \mu, \epsilon 1,340$ at $369 \mathrm{~m} \mu$.

\subsection{Outline of Method}

The principle of the spectrophotometric determination of a $p K$ value is as follows. Consider three solutions of a phenol, all at the same stoichiometric concentration. Let there be added to one enough acid to convert the phenol entirely into the molecular form, HN. To the second let there be added sufficient alkali to convert the phenol entirely into its ionized form, $\mathrm{N}^{-}$. Let the third contain a buffer solution in which the phenol is present partly in its molecular form and partly in its ionized form. Let $\alpha$ be the degree of dissociation of the phenol. Then if $D_{1}, D_{2}$, and $D$, respectively, are the optical densities of these three solutions, measured at the same wavelength in cells of the same length, it follows that

$$
\begin{aligned}
& \alpha=\left(D-D_{1}\right) /\left(D_{2}-D_{1}\right) \text { and } \\
& \alpha /(1-\alpha)=\left(D-D_{1}\right) /\left(D_{2}-D\right) .
\end{aligned}
$$

This dissociation constant for the process

$$
\mathrm{HN} \rightleftharpoons \mathrm{H}^{+}+\mathrm{N}^{-}
$$

is

$$
K=\frac{a_{\mathrm{H}^{+}} \cdot m_{\mathrm{N}^{-}}}{m_{\mathrm{HN}}} \frac{\gamma_{\mathrm{N}}^{-}}{\gamma_{\mathrm{HN}}}=a_{\mathrm{H}^{+}} \cdot \frac{\alpha}{1-\alpha} \frac{\gamma_{\mathrm{N}}^{-}}{\gamma_{\mathrm{HN}}}
$$

which can be written as

$$
p K=p\left(a_{\mathrm{H}} \gamma_{\mathrm{Cl}}\right)-\log \alpha /(1-\alpha)-\log \gamma_{\mathrm{N}^{-}} /\left(\gamma_{\mathrm{HN}} \gamma_{\mathrm{Cl}}{ }^{-}\right)
$$

where $p\left(a_{\mathrm{H}} \gamma_{\mathrm{Cl}}\right)$ is a characteristic of the buffer solution employed and $\gamma$ designates an activity coefficient.

Accurate values of the $p K$ are obtained if $\alpha \approx 0.5$, which means that $p K$ should be approximately equal to $p\left(a_{\mathrm{H}} \gamma_{\mathrm{Cl}}\right)$. Thus, the measurement of the $p K$ value of a phenol is best performed with a buffer solution which satisfies this condition. The buffer solutions used in this work were (1) an equimolal mixture of potassium dihydrogen phosphate and disodium hydrogen phosphate, $p\left(a_{\mathrm{H}} \gamma_{\mathrm{Cl}}\right) \approx 7.0$; (2) a mixture of tris(hydroxymethyl)aminomethane $(m)$ and its hydrochloride $(0.9845 m), p\left(a_{\mathrm{H}} \gamma_{\mathrm{Cl}}\right) \approx 8.3$; (3) a mixture of borax $(m)$ and sodium chloride $(1.855 m), p\left(a_{\mathrm{H}} \gamma_{\mathrm{Cl}}\right) \approx 9.2$. The $p\left(a_{\mathrm{H}} \gamma_{\mathrm{Cl}}\right)$ values of the buffer solutions depend on the concentrations of the buffer constituents; the requisite values have been tabulated by Bates and Gary [3]. These $p\left(a_{\mathrm{H}} \gamma_{\mathrm{Cl}}\right)$ values are altered to a small extent by the phenol in the buffer solution whose dissociation into hydrogen ions increases the acidity of the buffer solution slightly. The correction to the $p\left(a_{\mathrm{H}} \gamma_{\mathrm{Cl}}\right)$ values has already been considered [4].

It would be expected that the last term in eq (3) would be either small or negligible; in the former case an extrapolation can be made to give the true value of $p K$ at infinite dilution. For the phenols studied in this work, the value of $p K$ proved to be independent of the buffer concentration, indicating that the last term of eq (3) was negligible and, therefore, the best value of $p K$ was assumed to be the average of those found at different concentrations of the buffer solution.

The dissociation of the anilinium ion or a substituted anilinium ion can be represented as

$$
\mathrm{BH}^{+} \rightleftharpoons \mathrm{H}^{+}+\mathrm{B}
$$

with a dissociation constant

$$
K=\frac{m_{\mathrm{H}^{+}} \cdot \alpha}{1-\alpha} \frac{\gamma_{\mathrm{H}^{+}} \cdot \gamma_{\mathrm{B}}}{\gamma_{\mathrm{BH}^{+}}}
$$

or

$$
\begin{aligned}
p K=-\log m_{\mathrm{H}}^{+}-\log \alpha /( & 1-\alpha) \\
& -\log \left(\gamma_{\mathrm{H}^{+}} \cdot \gamma_{\mathrm{B}}\right) / \gamma_{\mathrm{BH}^{+}} .
\end{aligned}
$$

The $p K$ values of most substituted anilines are less than 3 and it is therefore preferable to make measurements in solutions of a strong acid of known concentration. In this work, hydrochloric acid was used. Then, if $m_{\mathrm{HCl}}$ is the stoichiometric concentration of hydrochloric acid added to the aniline solution and $m$ is the stoichiometric concentration of aniline, $m_{\mathrm{H}^{+}}$ in eq (5) can be equated to $m_{\mathrm{HCl}}-m(1-\alpha)$. Again it is desirable to use hydrochloric acid solutions of concentration such that $\alpha \approx 0.5$. If dilute solutions of hydrochloric acid are used, the last term of eq (5) should be negligible and this proved to be the case. But if $p K$ was small, so that moderately concentrated solutions of hydrochloric acid had to be used, as in the case of 2,6-dibromoaniline and 4-methyl-2-nitro- 
aniline, this term could no longer be neglected and the true value of $p K$ had to be obtained by extrapolation of $\left[-\log m_{\mathrm{H}^{+}}-\log \alpha /(1-\alpha)\right]$ to zero acid concentration; this procedure has already been used for 2,6-dichloroaniline [1] and for $o$-nitroaniline [2].

In one instance, 4-chloro-2-methylaniline, with a $p K$ value $\approx 3.8$, it was necessary to use a succinic acid-sodium hydrogen succinate buffer solution. The equation for the dissociation constant now becomes

$$
p K=p\left(a_{\mathrm{H}} \gamma_{\mathrm{Cl}}\right)-\log \alpha /(1-\alpha)+\log \left(\gamma_{\mathrm{BH}^{+}} \cdot \gamma_{\mathrm{Cl}^{-}}\right) / \gamma_{\mathrm{B}}
$$

Values of $p\left(a_{\mathrm{H}} \gamma_{\mathrm{Cl}}\right)$ for this buffer solution have been tabulated [3] and the activity coefficient term can be written

$$
\log \left(\gamma_{\mathrm{BH}^{+}} \cdot \gamma_{\mathrm{Cl}^{-}}\right) / \gamma_{\mathrm{B}}=-2 \mathrm{~A} I^{1 / 2} /\left(1+1.5 I^{1 / 2}\right)
$$

where $A$ is the parameter of the Debye-Hückel equation $\left(0.5108 \mathrm{~kg}^{-1 / 2} \mathrm{~mole}^{1 / 2}\right.$ at $\left.25{ }^{\circ} \mathrm{C}\right)$.

\section{Results and Discussion}

\subsection{Additivity Effects}

The detailed experimental results are given in tables $1-11$ and summarized in table 12 . The $p K$ value for 4-methyl-2-nitrophenol is taken from a previous publication [5]. Table 12 also contains values of $p K$ calculated on the assumption that the effect of double substitution in an aniline or phenol can be compounded as the sum of the separate monosubstitution effects. Thus the $p K$ value of phenol itself, 9.998, [2] is raised to $10.28_{7}$ in $o$-cresol, [6] the substitution effect of the $o$-methyl group being $-0.28_{9}$; the $p K$ value of $p$ chlorophenol is $9.41_{8}[2]$ so that the substitution effect of the p-chloro group is $0.58_{0}$. If these effects are strictly additive, the combined effects of $o$-methyl and $p$-chloro substitution should be $0.29_{1}$ giving $p K=9.70_{7}$ for 4-chloro-2-methylphenol, which agrees very well with the observed value of $9.70_{6}$. Substituent effects were calculated from the $p K$ values of $o$-nitrophenol [7], $m$-nitrophenol [8], and $m$-nitroaniline [10]; for other groups the data of Biggs [6] or of Biggs and Robinson [2] were used.

Inspection of table 12 shows that the additivity rule holds with varying degrees of success. It is remarkably good with 4-chloro-2-methylphenol and 4-methyl3-nitrophenol and not so good with 4-methyl-2-nitrophenol. It is possible that the additivity rule fails when bulky ortho substituted groups are present because these can produce steric inhibition of resonance. Thus the rule fails badly with 2,6-dibromoaniline and 2,6-dibromophenol as it does with the corresponding dichloro compounds [1] and with 2,6-dinitrophenol [10]. Perhaps the most remarkable failure of additivity is found with 4-methyl-2-nitroaniline; the additivity rule holds for the corresponding phenol with only moderate success.
TABLE 1. Dissociation constant of 4-chloro-2-methylaniline

Aniline concentration $2.27 \times 10^{-4} M$, cell length $1 \mathrm{~cm}$, wavelength $291 \mathrm{~m} \mu$, $D_{1} 0, D_{2} 0.569$

\begin{tabular}{r|r|r|r|r|r|r|r}
\hline$I$ & \multicolumn{1}{|c|}{$m$} & $p\left(a_{\mathrm{H}} \gamma_{\mathrm{Cl}}\right)$ & \multicolumn{1}{c|}{$D$} & $\log \frac{D-D_{1}}{D_{2}-D}$ & $2 \log \gamma$ & $p K$ & $p K($ corr $)$ \\
\hline .02017 & 0.02 & 3.898 & 0.257 & -0.084 & -0.117 & 3.865 & 3.853 \\
.03018 & .03 & 3.892 & .251 & -.103 & -.140 & 3.855 & 3.848 \\
.04019 & .04 & 3.887 & .245 & -.121 & -.157 & 3.851 & 3.848 \\
.05019 & .05 & 3.884 & .239 & -.140 & -.171 & 3.853 & 3.851 \\
.06020 & .06 & 3.882 & .236 & -.150 & -.183 & 3.849 & 3.847 \\
.08021 & .08 & 3.878 & .229 & -.172 & -.203 & 3.847 & 3.846 \\
.10021 & .10 & 3.875 & .223 & -.191 & -.219 & 3.847 & 3.846 \\
\hline
\end{tabular}

The buffer solution was a mixture of sodium hydrogen succinate $(m)$ and hydrochloric acid $(0.6667 \mathrm{~m})$ equivalent to a mixture of sodium hydrogen succinate $(0.3333 \mathrm{~m})$, succinic acid $(0.6667 \mathrm{~m})$, and sodium chloride $(0.6667 \mathrm{~m})$.

\begin{tabular}{|c|c|c|c|c|}
\hline \multicolumn{5}{|c|}{$\begin{array}{l}\text { Aniline concentration } 9.31 \times 10^{-5} M \text {, cell length } 4 \mathrm{~cm} \text {, } \\
\text { wavelength } 300 \mathrm{~m} \mu, D_{1} 0.009, D_{2} 0.804\end{array}$} \\
\hline$m_{\mathrm{HCl}} \times 10^{3}$ & $D$ & $\log \frac{D-D_{1}}{D_{2}-D}$ & $-\log m_{\mathrm{H}}{ }^{+}$ & $p K$ \\
\hline 5.846 & 0.568 & 0.375 & 2.235 & 1.860 \\
\hline 6.852 & .535 & $\begin{array}{r}.291 \\
.291\end{array}$ & 2.166 & 1.875 \\
\hline 9.591 & .474 & .149 & 2.020 & 1.871 \\
\hline 13.80 & .408 & .003 & 1.862 & 1.859 \\
\hline 16.28 & .370 & -.080 & 1.790 & 1.870 \\
\hline 16.39 & .375 & -.069 & 1.787 & 1.856 \\
\hline 21.02 & .322 & -.188 & 1.679 & 1.867 \\
\hline 29.59 & .257 & -.344 & 1.530 & 1.874 \\
\hline
\end{tabular}

TABLE 2. Dissociation constant of 2,4-dibromoaniline

\begin{tabular}{|c|c|c|c|c|c|}
\hline \multicolumn{6}{|c|}{$\begin{array}{c}\text { Aniline concentration } 4.92 \times 10^{-5} \mathrm{M} \text {, cell length } 4 \mathrm{~cm} \text {, } \\
\text { wavelength } 294 \mathrm{~m} \mu, D_{1} 0, D_{2} 0.502\end{array}$} \\
\hline$m_{\mathrm{HCl}}$ & $D$ & $\log \frac{D-D_{1}}{D_{22}-D}$ & $\log m_{\mathrm{H}^{+}}$ & $p K^{\prime}$ & $p K^{\prime}($ calc $)$ \\
\hline 0.1021 & 0.436 & 0.817 & -0.991 & 0.174 & 0.182 \\
\hline .2042 & .382 & .499 & -.690 & .191 & .195 \\
\hline .3063 & .337 & .306 & -.514 & .208 & .209 \\
\hline .4084 & .300 & .167 & -.389 & .222 & .222 \\
\hline .5105 & .271 & .065 & -.292 & .227 & .236 \\
\hline .6126 & .242 & -.037 & -.213 & .250 & .249 \\
\hline .7147 & .218 & -.121 & -.146 & .267 & .263 \\
\hline .8168 & .196 & -.200 & -.088 & .288 & .277 \\
\hline .9189 & .183 & -.248 & -.037 & .285 & .290 \\
\hline 1.021 & .165 & -.318 & .009 & .309 & .304 \\
\hline
\end{tabular}

TABLE 3. Dissociation constant of 2,6-dibromoaniline

$p K^{\prime}($ calc $)=0.168+0.133 m_{\mathrm{HCl}}$.

\begin{tabular}{|c|c|c|c|c|}
\hline$m_{\mathrm{HCl}} \times 10^{3}$ & $D$ & $\log \frac{D-D_{1}}{D_{22}-D}$ & $-\log m_{\mathrm{H}^{+}}$ & $p K$ \\
\hline 2.004 & 0.772 & 0.393 & 2.731 & 2.338 \\
\hline 4.007 & .613 & .074 & 2.424 & 2.350 \\
\hline 6.011 & .522 & -.096 & 2.242 & 2.338 \\
\hline 8.014 & .451 & -.232 & 2.114 & 2.346 \\
\hline 10.02 & .403 & -.331 & 2.014 & 2.345 \\
\hline 14.03 & .336 & -.485 & 1.865 & 2.350 \\
\hline
\end{tabular}

TABLE 4. Dissociation constant of 2-methyl-5-nitroaniline 
TABLE 5. Dissociation constant of 4-methyl-2-nitroaniline

\begin{tabular}{|c|c|c|c|c|c|}
\hline \multicolumn{6}{|c|}{$\begin{array}{c}\text { Aniline concentration } 1.752 \times 10^{-4} M \text {, cell length } 1 \mathrm{~cm} \text {, } \\
\text { wavelength } 427 \mathrm{~m} \mu, D_{1} 0, D_{2} 0.740\end{array}$} \\
\hline$m_{\mathrm{HCl}}$ & $D$ & $\log \frac{D-D_{1}}{D_{2}-D}$ & $-\log m_{\mathrm{H}^{+}}$ & $p K^{\prime}$ & $p K^{\prime}($ calc $)$ \\
\hline 0.1037 & 0.581 & 0.563 & 0.984 & 0.421 & 0.422 \\
\hline .2075 & .471 & .243 & .683 & .440 & .442 \\
\hline .3112 & .390 & .047 & .507 & .460 & .461 \\
\hline .4150 & .326 & -.104 & .382 & .486 & .480 \\
\hline .5187 & .280 & -.216 & .285 & .501 & .499 \\
\hline .6224 & .243 & -.311 & .206 & .517 & .519 \\
\hline .7262 & .210 & -.402 & .139 & .541 & .538 \\
\hline .8299 & .185 & -.477 & .081 & .558 & .557 \\
\hline .9337 & .163 & -.549 & .030 & .579 & .577 \\
\hline 1.037 & .146 & -.609 & -.016 & .593 & .596 \\
\hline
\end{tabular}

$p K^{\prime}($ calc $)=0.403+0.186 m_{\mathrm{HCl}}$.

TABLE 6. Dissociation constant of 4-methyl-3-nitroaniline

Aniline concentration $3.49 \times 10^{-4} M$, cell length $1 \mathrm{~cm}$, wavelength $357 \mathrm{~m} \mu, D_{1} 0.088, D_{2} 0.551$

\begin{tabular}{r|r|r|r|r}
\hline$m_{\mathrm{HCl}} \times 10^{3}$ & \multicolumn{1}{|c|}{$D$} & $\log \frac{D-D_{1}}{D_{2}-D}$ & $-\log m_{\mathrm{H}^{+}}$ & $p K$ \\
\hline & & & & \\
\hline 1.002 & 0.336 & +0.062 & 3.076 & 3.014 \\
1.503 & .284 & -.134 & 2.885 & 3.019 \\
2.004 & .250 & -.269 & 2.750 & 3.019 \\
2.505 & .225 & -.377 & 2.646 & 3.023 \\
3.006 & .207 & -.461 & 2.561 & 3.022 \\
3.507 & .193 & -.533 & 2.490 & 3.027 \\
4.008 & .181 & -.600 & 2.428 & 3.028 \\
5.010 & .165 & -.700 & 2.326 & 3.026 \\
\hline
\end{tabular}

TABLE 7. Dissociation constant of 4-chloro-2-methylphenol

\begin{tabular}{|c|c|c|c|c|c|}
\hline \multicolumn{6}{|c|}{$\begin{array}{c}\text { Pिenol concentrution } 3.25 \times 10^{-4} M \text {, cell length } 1 \mathrm{~cm} \text {, } \\
\text { wavelength } 300 \mathrm{~m} \mu, D_{1} 0.012, D_{2} 0.984\end{array}$} \\
\hline$I$ & $p\left(a_{\mathrm{H}} \gamma_{\mathrm{Cl}}\right)$ & $D$ & $\log \frac{D-D_{1}}{D_{2}-D}$ & $p K$ & $p K($ corr $)$ \\
\hline 0.010 & 9.234 & 0.254 & -0.480 & 9.714 & 9.702 \\
\hline .015 & 9.237 & .257 & -.472 & 9.709 & 9.701 \\
\hline .020 & 9.237 & .258 & -.470 & 9.707 & 9.701 \\
\hline .025 & 9.238 & .257 & -.472 & c. 710 & 9.705 \\
\hline .030 & 9.239 & .256 & -.475 & 9.714 & 9.710 \\
\hline .035 & 9.239 & .256 & -.475 & 9.714 & 9.711 \\
\hline .040 & 9.239 & .256 & -.475 & 9.714 & 9.711 \\
\hline
\end{tabular}

The buffer solution was a mixture of borax $(m)$ and sodium chloride $(1.855 \mathrm{~m}), I=3.855 \mathrm{~m}$.
TABLE 8. Dissociation constant of 2,4-dibromophenol

\begin{tabular}{r|r|r|r|r|r}
\hline \hline \multicolumn{6}{c}{$\begin{array}{l}\text { Phenol concentration } 1.76 \times 10^{-4} M, \text { cell length } 1 \mathrm{~cm}, \\
\text { wavelength } 309 \mathrm{~m} \mu, D_{1} 0.007, D_{2} 0.652\end{array}$} \\
\hline & & & & & \\
\hline & $p\left(a_{\mathrm{H}} \gamma_{\mathrm{Cl}}\right)$ & $D$ & $\log \frac{D-D_{1}}{D_{2}-D}$ & $p K$ & $p K$ (corr) \\
& & & & & \\
\hline & & & & & \\
0.01 & 8.176 & 0.461 & 0.376 & 7.800 & 7.790 \\
.02 & 8.207 & .471 & .409 & 7.798 & 7.793 \\
.03 & 8.232 & .480 & .439 & 7.793 & 7.790 \\
.04 & 8.251 & .486 & .460 & 7.791 & 7.789 \\
.05 & 8.266 & .491 & .478 & 7.788 & 7.786 \\
.06 & 8.280 & .495 & .492 & 7.788 & 7.786 \\
.07 & 8.292 & .499 & .507 & 7.785 & 7.784 \\
.08 & 8.302 & .499 & .507 & 7.795 & 7.794 \\
.09 & 8.312 & .502 & .518 & 7.794 & 7.793 \\
.10 & 8.321 & .505 & .530 & 7.791 & 7.790 \\
\hline
\end{tabular}

The buffer solution was a mixture of tris(hydroxymethyl)amino methane $(m)$ and its hydrochloride $(0.9845 \mathrm{~m}), I=0.9845 \mathrm{~m}$.

TABLE 9. Dissociation constant of 2,6-dibromophenol

Phenol concentration $3.05 \times 10^{-5} M$, cell length $4 \mathrm{~cm}$, wavelength $300 \mathrm{~m} \mu, D_{1} 0.008, D_{2} 0.641$

\begin{tabular}{r|r|r|r|r|r}
\hline \multicolumn{1}{l|}{} & $p\left(a_{\mathrm{H}} \gamma_{\mathrm{Cl}}\right)$ & \multicolumn{1}{c|}{$D$} & $\log \frac{D-D_{1}}{D_{2}-D}$ & $p K$ & $p K$ (corr) \\
\hline .01 & 7.111 & 0.467 & 0.421 & 6.690 & 6.684 \\
.02 & 7.080 & .463 & .408 & 6.672 & 6.669 \\
.03 & 7.058 & .456 & .384 & 6.674 & 6.672 \\
.04 & 7.040 & .452 & .371 & 6.669 & 6.667 \\
.05 & 7.026 & .443 & .342 & 6.684 & 6.683 \\
.06 & 7.013 & .444 & .345 & 6.668 & 6.667 \\
.08 & 6.992 & .433 & .310 & 6.682 & 6.681 \\
.10 & 6.974 & .432 & .307 & 6.667 & 6.666 \\
\hline
\end{tabular}

The buffer solution was an equimolal mixture of potassium dihydrogen phosphate $(m)$ and disodium hydrogen phosphate $(m)$, $I=4 m$.

TABLE 10. Dissociation constant of 2-methyl-5-nitrophenol

\begin{tabular}{l}
\hline \multicolumn{6}{c}{$\begin{array}{l}\text { Phenol concentration } 4.04 \times 10^{-4} M, \text { cell length } 1 \mathrm{~cm}, \\
\text { wavelength } 430 \mathrm{~m} \mu, D_{1} 0.005, D_{2}\end{array} .685$} \\
\hline \\
\hline
\end{tabular}

The buffer solutions were mixtures of tris(hydroxymethyl)amino methane $(m)$ and its hydrochloride $(0.9845 m), I=0.9845 m$. 
TABLE 11. Dissociation constant of 4-methyl-3-nitrophenol

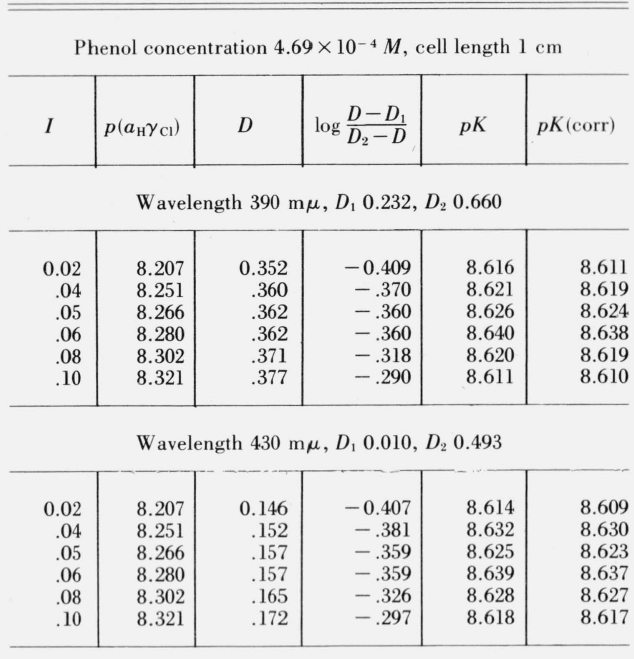

The buffer solutions were mixtures of tris(hydroxymethyl)amino methane $(m)$ and its hydrochloride $(0.9845 m), I=0.9845 m$.

TABLE 12. $\mathrm{pK}$ values of substituted anilines and phenols in aqueous solution at $25^{\circ} \mathrm{C}$

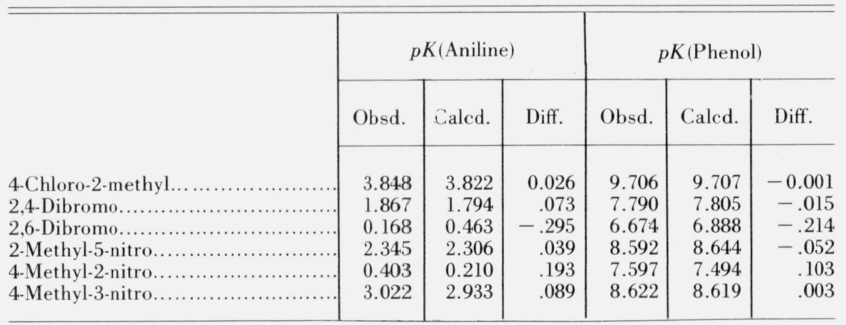

\subsection{A Linear Relation Between the $p K$ Values of Substituted Anilines and Those of Substituted Phenols}

Figure 1 is a plot of the $p K$ values of 25 substituted anilines $\left(p K_{A}\right)$ against the $p K$ value of the corresponding substituted phenol $\left(p K_{P}\right)$. It is clear that, with six exceptions, the experimental values lie close to a straight line. The exceptions are $o$-methyl, $p$-methoxy, $o$-nitro, 4-chloro-2-methyl, 2-methyl-5-nitro, and 4methyl-2-nitroaniline and phenol. The best straight line through the remaining 19 points in figure 1 , determined by the method of least squares, is given by the equation:

$$
p K_{A}=a p K_{P}-b,
$$

with $a=1.293$ and $b=8.254$. This equation is essentially an expression of the Hammett Rule [11]. It enables the $p K$ value of a substituted aniline to be predicted from the $p K$ value of the corresponding phenol (or vice versa) with an average difference between observed and calculated values of $0.07_{7}$, the smallest difference being found in the case of $p$-nitro substitution $\left(0.00_{1}\right)$ and the greatest in the case of

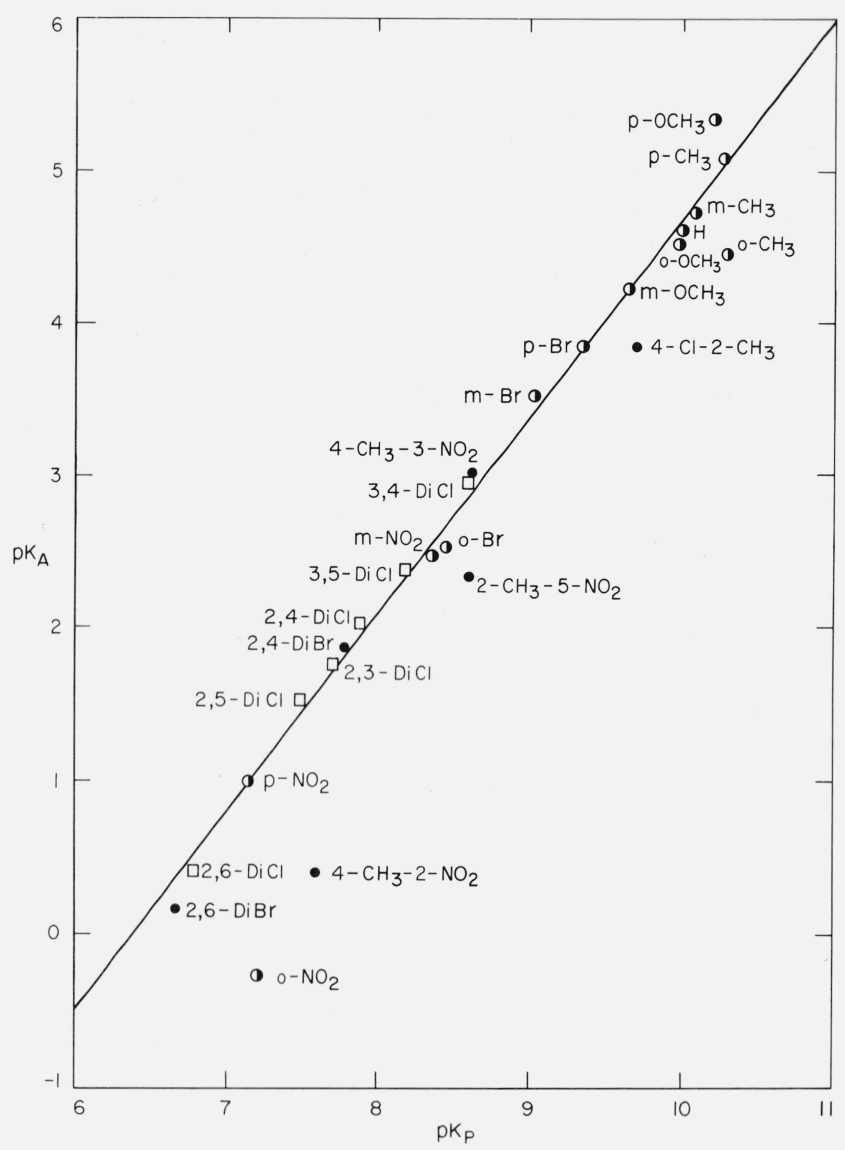

FIGURE 1. $\mathrm{pK}_{\mathrm{A}}$ values of substituted anilines versus $\mathrm{pK}_{\mathrm{P}}$ values of substituted phenols.

D, Monosubstituted compounds

, Dichloro compounds

, Present work.

2,6-dibromo substitution $\left(0.20_{6}\right)$. In a previous publication [1], a similar linear relationship was discussed but two linear equations were proposed, one for the monosubstituted anilines and phenols and another for dichloroanilines and dichlorophenols. Naturally, the use of four parameters leads to better agreement with the experimental data; a two-parameter equation is used here because it is not so much agreement with the experimental data that is of interest but the exceptional cases which are widely divergent. The exceptions among the monosubstituted anilines and phenols are $o$-methyl, $p$-methoxy, and $o$-nitro compounds for which deviations from the straight line of figure 1 of $0.59_{8},-0.39_{8}$, and $1.35_{9}$, respectively, are found, a positive sign being used if the observed $p K_{A}$ value of a substituted aniline is greater than that calculated using eq (8). Three exceptions are found among the disubstituted anilines and phenols, the 4-chloro-2-methyl, 2-methyl-5-nitro, and 4-methyl-2nitroanilines and phenols. The discrepancies amount to $0.44_{8}, 0.51_{0}$, and $1.16_{6}$, respectively. It will be noted that there is $o$-methyl substitution in two instances and $o$-nitro substitution in the third. Both substituents lead to anomalies in the monosubstituted 
compounds. Moreover, the anomaly is about the same in both monosubstituted and disubstituted compounds. Thus it is $0.59_{8}$ for $o$-methyl monosubstitution and $0.44_{8}$ or $0.51_{0}$ if there is an $o$-methyl radical in a disubstituted compound; it is 1.359 for $o$-nitro monosubstitution and $1.16_{6}$ if there is an $o$-nitro group in a disubstituted compound. This suggests that anomalies in monosubstituted compounds carry over more or less unchanged on further substitution by a "wellbehaved" radical. On the average, the anomaly is $0.51_{9}$ for $o$-methyl substitution and $1.26_{2}$ for $o$-nitrosubstitution; eq (8) can, therefore, be extended by retaining the value of $a=1.293$ but reducing $b$ to -9.516 for $o$-nitro substitution and $b$ to -8.773 for $o$-methyl substitution. The agreement between observed $p K_{A}$ values and those calculated with the aid of these $b$ values in eq (8) is now $-0.09_{5}$ for $o$-nitro monosubstitution and -0.079 for $o$-methyl monosubstitution; in the case of disubstituted compounds, the discrepancy is $0.09_{6}$ for the 4-methyl-2-nitro compounds, $0.07_{1}$ for the 4-chloro-2-methyl compounds, and $0.00_{9}$ for the 2-methyl-5-nitro compounds.

\section{References}

[1] R. A. Robinson, J. Res. NBS 68A (Phys. and Chem.) No. 2, 159 (1964).

[2] A. I. Biggs and R. A. Robinson, J. Chem. Soc. (London) 388 (1961).

[3] R. G. Bates and R. Gary, J. Res. NBS 65A (Phys. and Chem.) No. 6, 495 (1961)

[4] R. A. Robinson and A. K. Kiang, Trans. Faraday Soc. 51, 1398 (1955).

[5] R. A. Robinson and R. G. Bates, J. Res. NBS 70A (Phys. and Chem.) No. 6, 553 (1966).

[6] A. I. Biggs, Trans. Faraday Soc. 52, 35 (1956).

[7] R. A. Robinson and A. Peiperl, J. Phys. Chem. 67, 1723 (1963).

[8] R. A. Robinson and A. Peiperl, J. Phys. Chem. 67, 2860 (1963).

[9] E. E. Sager, R. A. Robinson, and R. G. Bates, J. Res. NBS 68A (Phys. and Chem.) No. 3, 305 (1964).

[10] R. A. Robinson, M. M. Davis, M. Paabo, and V. E. Bower, J. Res. NBS 64A (Phys. and Chem.) No. 4, 347 (1960).

[11] L. P. Hammett, Physical organic chemistry, ch. VII (McGrawHill Book Co., Inc., New York, N.Y., 1940).

(Paper 71A3-453) 